\title{
Soluble Programmed Cell Death-1 Predicts Hepatocellular Carcinoma Development During Nucleoside Analogue Treatment
}

\section{Ritsuzo Kozuka}

Osaka City University

Masaru Enomoto ( $\nabla$ enomoto-m@med.osaka-cu.ac.jp )

Osaka City University

Minh Phuong Dong

Osaka City University

Hoang Hai

Osaka City University

Le Thi Thanh Thuy

Osaka City University

Naoshi Odagiri

Osaka City University

Kanako Yoshida

Osaka City University

Kohei Kotani

Osaka City University

Hiroyuki Motoyama

Osaka City University

Etsushi Kawamura

Osaka City University

Atsushi Hagihara

Osaka City University

Hideki Fujii

Osaka City University

Sawako Uchida-Kobayashi

Osaka City University

Akihiro Tamori

Osaka City University

Norifumi Kawada

Osaka City University 


\section{Research Article}

Keywords: Hepatocellular carcinoma, soluble programmed cell death-1, a-fetoprotein

Posted Date: August 17th, 2021

DOl: https://doi.org/10.21203/rs.3.rs-793073/v1

License: (c) (i) This work is licensed under a Creative Commons Attribution 4.0 International License. Read Full License

Version of Record: A version of this preprint was published at Scientific Reports on January 7th, 2022. See the published version at https://doi.org/10.1038/s41598-021-03706-w. 


\section{Abstract}

Soluble immune checkpoint molecules are emerging novel mediators of immune regulation. However, it is unclear whether soluble immune checkpoint proteins affect the development of hepatocellular carcinoma (HCC) during nucleos(t)ide analogue (NA) treatment in patients with chronic hepatitis B virus infection. This study included 122 NA-naïve patients who received NA therapy. We assessed the associations of clinical factors, including soluble immune checkpoint proteins, with HCC development during NA treatment. The baseline serum concentrations of 16 soluble immune checkpoint proteins were measured using multiplexed fluorescent bead-based immunoassay. In total, 13 patients developed HCC during the follow-up period (median duration, 4.3 years). Of the 16 proteins, soluble inducible T-cell co-stimulator ( $\geq 164.71 \mathrm{pg} / \mathrm{mL} ; p=0.014)$, soluble programmed cell death-1 (sPD-1) ( $\leq 447.27 \mathrm{pg} / \mathrm{mL} ; p=0.031)$, soluble CD40 ( $\leq 493.68 \mathrm{pg} / \mathrm{mL} ; p=0.032)$, and soluble herpes virus entry mediator $(\leq 2,470.83 \mathrm{pg} / \mathrm{mL}$; $p=0.038$ ) were significantly associated with HCC development (log-rank test). In a multivariate analysis, an sPD-1 level $\leq 447.27 \mathrm{pg} / \mathrm{mL}(p=0.014$; hazard ratio [HR], 4.537) and a-fetoprotein level $\geq 6.4 \mathrm{ng} / \mathrm{mL}$ $(p=0.040 ; \mathrm{HR}, 5.524)$ were independently and significantly associated with HCC development. SPD- 1 is a novel predictive biomarker for HCC development during NA treatment.

\section{Introduction}

Hepatitis B virus (HBV) infection is an important public health issue and a leading cause of cirrhosis and hepatocellular carcinoma (HCC), being responsible for more than 686,000 deaths annually. ${ }^{1}$

Nucleos(t)ide analogues (NAs) such as entecavir and tenofovir are widely used in patients with chronic $\mathrm{HBV}$ infection. Moreover, NA treatment for chronic HBV infection has been reported to not only suppress HBV replication but also reduce the risk of HCC development. ${ }^{2-4}$ However, some patients still develop HCC despite effective treatment with NA, especially when HCC-related risk factors are reported during the natural course. ${ }^{3-7}$ We reported that human leukocyte antigen -DQA1/DRB1 polymorphism and cirrhosis were risk factors for $\mathrm{HCC}$ development during entecavir treatment. ${ }^{8}$ Therefore, understanding the HCCrisked factors before the start of NA treatment may improve HCC surveillance in patients with chronic HBV infection.

The risk factors for developing HCC can be classified as host, viral, and environmental factors. In particular, it is important to investigate host immunological factors for HCC development because immune dysregulation with the exhaustion of HBV-specific CD8 + T cells plays an important role in persistent HBV infection and contributes to the immunopathogenesis of HBV-associated liver diseases, including hepatocarcinogenesis. ${ }^{9-17}$

Circulating soluble immune checkpoint proteins, members of a family of full-length receptors produced via mRNA expression or the cleavage of membrane-bound proteins, have been studied as host immunological factors in various cancers. ${ }^{18}$ Moreover, these soluble immune checkpoint proteins can diffuse in the serum. However, the functions of these proteins are unclear; soluble forms of inhibitory 
factors are not necessarily involved in negative immune regulation, and vice versa. Soluble immune checkpoint proteins, such as soluble B- and T-lymphocyte attenuator (sBTLA), ${ }^{19}$ soluble T-cell immunoglobulin and mucin domain-3 (sTIM-3), ${ }^{20}$ soluble herpes virus entry mediator (sHVEM), ${ }^{21}$ soluble programmed cell death-1 (sPD-1), ${ }^{22-24}$ soluble cytotoxic T-lymphocyte associated antigen 4 (sCTLA-4), ${ }^{25}$ and soluble programmed cell death-ligand 1 (SPD-L1), ${ }^{24,26,27}$ are linked to the development and prognosis of HCC, and are potential biomarkers and therapeutic targets.

However, it is unclear whether soluble immune checkpoint proteins affect HCC development during NA treatment in patients with chronic HBV infection. Therefore, we evaluated host immunological factors associated with HCC development, i.e., soluble immune checkpoint proteins, during NA treatment.

\section{Patients And Methods}

\section{Patients}

Of 127 patients in our previous study, 122 patients for whom stored serum samples collected before the start of entecavir treatment were available were included in this retrospective study. These 122 NA-naïve patients with chronic HBV infection, verified as HBsAg-positive and HBV DNA-positive for at least 6 months before treatment, underwent entecavir therapy from September 2006 to September 2016 at Osaka City University Hospital. The inclusion criteria were as follows: persistent elevation of the serum ALT level ( $\geq 31 \mathrm{U} / \mathrm{L}$ ) and the HBV DNA level ( $\geq 4.0 \log$ copies/mL; equivalent to $3.3 \log \mathrm{IU} / \mathrm{mL}$ ) as well as the presence of advanced fibrosis even if the ALT level was within the normal range according to published guidelines, ${ }^{1,38,39}$ no clinical signs of HCC before the start of entecavir treatment, and no evidence of hepatitis $\mathrm{C}$ virus or human immunodeficiency virus co-infection and other likely causes of chronic liver disease.

The procedures were performed in accordance with the Helsinki Declaration of 1964 (2013 revision) and approved by the Ethics Committee of Osaka City University Hospital (no. 1646 and 3260). Written informed consent was obtained from each patient.

\section{Study design}

All patients were treated with entecavir for more than 1 year. Entecavir (Baraclude; Bristol-Myers, Tokyo, Japan) was given orally at a dose of $0.5 \mathrm{mg}$ once daily. During the follow-up period, clinical, biochemical, and HBV serological assessments were performed at 1-3-month intervals. Cirrhosis was diagnosed based on a histological examination grade of F4 according to the METAVIR scoring system, ${ }^{40}$ using imaging modalities such as ultrasonography, computed tomography (CT), or magnetic resonance imaging (MRI), and based on signs of portal hypertension.

\section{Patient evaluation}


Based on our previous study, ${ }^{8}$ we evaluated patients included in this study. The study endpoint was HCC development during entecavir treatment. Patients who developed HCC within 1 year after the start of treatment were excluded. All patients underwent ultrasonography or dynamic CT and/or MRI every 3-6 months for HCC surveillance. HCC was diagnosed based on the presence of arterial hypervascularization and delayed washout on dynamic CT and/or MRI. Patients were followed up until a diagnosis of HCC was confirmed or the last visit before December 2019.

\section{Laboratory assays}

Complete blood counts and serum aspartate aminotransferase (AST), ALT, $\gamma$-glutamyltransferase, total bilirubin, and albumin levels were determined using standard procedures. Serum AFP levels were determined via chemiluminescent enzyme immunoassay. Serum concentrations of hyaluronic acid were measured using latex agglutination immunoturbidimetry (Fujirebio Inc., Tokyo, Japan). The serum type IV collagen concentration was measured using latex agglutination turbidimetry (Daiichi Fine Chemical Co., Ltd., Tokyo, Japan). The FIB-4 index was calculated using Sterling's formula as age (years) $\times$ AST $(\mathrm{U} / \mathrm{L})$, platelet count $\left(\times 10^{9} / \mathrm{L}\right) \times \sqrt{ } \mathrm{ALT}(\mathrm{U} / \mathrm{L})$.

HBsAg was measured via chemiluminescent microparticle immunoassay (Architect HBsAg QT; Abbott Japan Corp., Tokyo, Japan). Hepatitis B e antigen (HBeAg) and anti-HBe were detected via chemiluminescent enzyme immunoassay. HBV DNA was measured using real-time polymerase chain reaction analysis (COBAS TaqMan HBV Test, ver. 2.0; Roche Diagnostics K.K., Tokyo, Japan). The HBV genotype was determined via enzyme-linked immunosorbent assay using monoclonal antibodies against type-specific epitopes in the preS2-region (Institute of Immunology, Tokyo, Japan). Mutations at nucleotide 1,896 in the precore region and nucleotides 1,762 and 1,764 in the basal core promoter region of HBV DNA were identified via enzyme-linked minisequencing assay (Genome Science Laboratory, Tokyo, Japan). These laboratory assays are similar to those reported in our previous study. ${ }^{8}$

\section{Soluble immune checkpoint protein assays}

Serum samples were collected at baseline. The serum levels of 16 soluble immune checkpoint proteins were measured via multiplexed fluorescent bead-based immunoassay using the Milliplex Map Kit (EMD Millipore Corporation, Danvers, MA, USA) and the Bio-Rad Luminex Bio-Plex- 200 system (Hercules, CA, USA). The 16 soluble proteins of interest were sBTLA, sCD27, sCD28, sTIM-3, sHVEM, sCD40, sGITR, sLAG-3, sTLR-2, sGITRL, SPD-1, sCTLA-4, sCD80, sCD86, sPD-L1, and sICOS. In accordance with the manufacturer's instructions, $12.5 \mu \mathrm{L}$ of serum was used for each measurement and all samples were assayed in duplicate; mean values were used for further analysis. For values below the limit of detection, we used $10 \%$ of the lowest recorded value as a substitute. ${ }^{19,41}$

In addition, of 13 patients who developed HCC during entecavir treatment, 9 had stored serum samples collected at 6 and 12 months after the start of entecavir treatment, at 6 and 12 months before HCC development, and at the time of HCC development, in which the SPD-1 level was measured via multiplexed fluorescent bead-based immunoassay. 


\section{Statistical analysis}

Statistical analyses were performed using JMP software (ver. 12.0; SAS Institute, Cary, NC, USA). Continuous variables were compared using the Mann-Whitney $U$ test, and discontinuous variables using Fisher's exact test. Receiver-operator curves were generated to obtain the optimal cut-off value for distinguishing between patients with and without HCC. Kaplan-Meier analysis and the log-rank test were used to analyze cumulative rates of HCC development. Cox proportional hazards models were used to identify factors associated with HCC development. Variables with $p$-values $<0.05$ in univariate Cox regression analyses were subjected to stepwise multivariate Cox regression analysis. The significance of changes in values between two time points was evaluated using the Wilcoxon signed-rank test. The significance of correlations was evaluated in Spearman's rank analysis. In two-tailed tests, $p<0.05$ was taken to indicate statistical significance.

\section{Results}

\section{Baseline characteristics}

Table 1 summarizes the baseline characteristics of the patients. The median (range) age was 45 (23-79) years, and there were $78(63.9 \%)$ males and $22(18.0 \%)$ patients with cirrhosis. A total of $104(85.2 \%)$ patients had HBV genotype $C$. Although the baseline characteristics were similar to those reported in our previous study, ${ }^{8}$ the median follow-up duration was extended from 4.4 (range, 1.0-10.7) to 6.2 (range, $1.1-13.3)$ years.

\section{Cumulative rates of HCC development according to clinical factors at baseline}

In our previous report, ${ }^{8} 10$ patients developed HCC during the follow-up period (median duration, 3.3 [range, 1.1-6.9] years); since then, an additional 3 patients have developed HCC (median duration, 4.3 [range, 1.1-7.6] years) (Table 2). In accordance with our previous report, ${ }^{8}$ liver fibrosis, the platelet count, the fibrosis-4 (FIB-4) index, age, type IV collagen, and a-fetoprotein (AFP) were significantly associated with HCC development according to the log-rank test. Moreover, hyaluronic acid and hepatitis $B$ surface antigen (HBsAg) were associated with HCC development (Supplementary Fig. 1).

\section{Cumulative rates of HCC development according to soluble immune checkpoint protein levels at baseline}

The cumulative rates of HCC development according to the serum soluble inducible T-cell co-stimulator (sICOS) level are shown in Fig. 1A. The cumulative rates of HCC development at 3, 5, 7, and 10 years were $9.5 \%, 20.3 \%, 24.1 \%$, and $27.9 \%$, respectively, in patients with sICOS $\geq 164.71 \mathrm{pg} / \mathrm{mL}(n=33)$, and $2.6 \%$, $4.3 \%, 8.1 \%$, and $8.1 \%$, respectively, in those with sICOS $<164.71 \mathrm{pg} / \mathrm{mL}(n=89)(p=0.014)$.

The cumulative rates of HCC development according to the serum SPD-1 level are shown in Fig. 1B. The cumulative rates of HCC development at $3,5,7$, and 10 years were $10.9 \%, 13.6 \%, 21.0 \%$, and $25.7 \%$, 
respectively, in patients with sPD-1 $\leq 447.27 \mathrm{pg} / \mathrm{mL}(n=51)$, and $0 \%, 5.9 \%, 8.0 \%$, and $8.0 \%$, respectively, in those with sPD-1 $>447.27 \mathrm{pg} / \mathrm{mL}(n=71)(p=0.031)$.

The cumulative rates of HCC development according to the serum soluble (s) CD40 level are shown in Fig. 1C. The cumulative rates of HCC development at 3, 5, 7, and 10 years were 8.6\%, 12.5\%, 19.1\%, and $21.9 \%$, respectively, in patients with $\operatorname{sCD} 40 \leq 493.68 \mathrm{pg} / \mathrm{mL}(n=64)$, and $0 \%, 5.4 \%, 5.4 \%$, and $5.4 \%$, respectively, in those with $\mathrm{SCD} 40>493.68 \mathrm{pg} / \mathrm{mL}(n=58)(p=0.032)$.

The cumulative rates of HCC development according to the serum sHVEM level are shown in Fig. 1D. The cumulative rates of HCC development at $3,5,7$, and 10 years were $5.3 \%, 11.7 \%, 23.1 \%$, and $27.4 \%$, respectively, in patients with sHVEM $\leq 2,470.83 \mathrm{pg} / \mathrm{mL}(n=43)$, and $4.2 \%, 7.7 \%, 7.7 \%$, and $7.7 \%$, respectively, in those with sHVEM $>2,470.83 \mathrm{pg} / \mathrm{mL}(n=79)(p=0.038)$.

There were no significant associations between HCC development and the levels of soluble immune checkpoint proteins (sBTLA, SCD27, sCD28, sTIM-3, soluble glucocorticoid-induced TNFR-related [sGITR], soluble lymphocyte-activation gene 3 [sLAG-3], soluble toll-like receptor 2 [sTLR-2], soluble glucocorticoidinduced TNFR-related ligand [sGITRL], sCTLA-4, sCD80, sCD86, and SPD-L1) (Supplementary Fig. 2).

\section{Predictive factors for HCC development during entecavir treatment}

As we reported previously, ${ }^{8}$ the FIB-4 index, platelet count, cirrhosis status, age, and AFP were associated with HCC development during entecavir treatment in univariate analyses. In addition, levels of type IV collagen $\geq 200 \mathrm{ng} / \mathrm{mL}$ ( $p=0.011$; hazard ratio [HR], 5.300; 95\% confidence interval [Cl], 1.458-19.270), hyaluronic acid $\geq 143 \mathrm{ng} / \mathrm{mL}(p=0.017 ; \mathrm{HR}, 3.922 ; 95 \% \mathrm{Cl}, 1.281-12.008), \mathrm{HBsAg} \leq 3.53 \mathrm{log} \mathrm{IU} / \mathrm{mL}(p=$ 0.027 ; HR, $5.507 ; 95 \% \mathrm{Cl}, 1.220-24.859)$, sICOS $\geq 164.71 \mathrm{pg} / \mathrm{mL}(p=0.022 ; \mathrm{HR}, 3.713 ; 95 \% \mathrm{Cl}, 1.210-$ 11.393), sPD-1 $\leq 447.27 \mathrm{pg} / \mathrm{mL}(p=0.042 ; \mathrm{HR}, 3.393 ; 95 \% \mathrm{Cl}, 1.044-11.033)$, and sHVEM $\leq 2,470.83$ $\mathrm{pg} / \mathrm{mL}(p=0.049 ; \mathrm{HR}, 3.078 ; 95 \% \mathrm{Cl}, 1.006-9.416)$ were significantly associated with HCC development during entecavir treatment (Table 3 ).

From multivariate analysis, a serum sPD-1 level $\leq 447.27 \mathrm{pg} / \mathrm{mL}(p=0.014 ; \mathrm{HR}, 4.537 ; 95 \% \mathrm{Cl}, 1.363-$ $15.103)$ and AFP level $\geq 6.4 \mathrm{ng} / \mathrm{mL}(p=0.040 ; \mathrm{HR}, 5.524 ; 95 \% \mathrm{Cl}, 1.084-28.164)$ were independently and significantly associated with HCC development during entecavir treatment (Table 3).

\section{Cumulative rates of HCC development according to the combination of serum SPD-1 and AFP levels}

When classified according to serum SPD-1 and AFP levels, the 10-year cumulative rates of HCC development were $0 \%, 6.5 \%, 12.8 \%$, and $67.0 \%$ in the sPD-1 $>447.27 \mathrm{pg} / \mathrm{mL}+$ AFP $<6.4 \mathrm{ng} / \mathrm{mL}$ group ( $n$ $=30$ ), sPD-1 $\leq 447.27 \mathrm{pg} / \mathrm{mL}+$ AFP $<6.4 \mathrm{ng} / \mathrm{mL}$ group ( $n=34), \mathrm{sPD}-1>447.27 \mathrm{pg} / \mathrm{mL}+$ AFP $\geq 6.4$ $\mathrm{ng} / \mathrm{mL}$ group $(n=41)$, and SPD-1 $\leq 447.27 \mathrm{pg} / \mathrm{mL}+$ AFP $\geq 6.4 \mathrm{ng} / \mathrm{mL}$ group $(n=17)$, respectively $(p<$ 0.0001) (Fig. 2).

Relationships between the serum SPD-1 level and clinical/virological characteristics 
The serum SPD-1 level at baseline was positively correlated with the HBV-DNA $(p=0.057, r=0.17$; Fig. 3A) and alanine aminotransferase (ALT) $(p<0.0001, r=0.41$; Fig. 3B) levels, and was similar between non-cirrhotic and cirrhotic patients (median 519.35 pg/mL vs. 534.62 pg/mL, $p=0.93$; Fig. 3C).

\section{Changes in serum SPD-1 level in patients who developed HCC during entecavir treatment}

We analyzed the changes in serum sPD-1 level in 9 (cases 1, 2, 3, 7, 8, 9, 11, 12, and 13; Table 2) of 13 patients who developed HCC during entecavir treatment (Fig. 4). The serum SPD-1 level decreased rapidly after 6 months of entecavir treatment. Significant decreases were found at $6(p=0.028)$ and $12(p=$ $0.028)$ months after the start of entecavir treatment, at $12(p=0.028)$ and $6(p=0.018)$ months before HCC development, and at the time of HCC development $(p=0.0077)$, compared with baseline values. Moreover, no re-increase in the serum SPD-1 level at HCC development was observed.

\section{Discussion}

To our knowledge, this is the first evaluation of the associations between soluble immune checkpoint proteins and HCC development during NA treatment in patients with chronic HBV infection. Our results indicate that SPD-1 and AFP are predictive of HCC development during NA treatment, and the predictive accuracy is increased using a combination of serum sPD-1 and AFP levels (Fig. 2).

Soluble immune checkpoint proteins, such as sBTLA, sTIM-3, sHVEM, sPD-1, sCTLA-4, and sPD-L1, may be predictive of the treatment response and prognosis in patients with HCC. ${ }^{19-27}$ However, the associations between these proteins and HCC development during NA treatment were unknown. In this study, we measured the concentrations of 16 soluble immune checkpoint proteins in the serum of patients treated with NAs, and detected a significant association between the serum sPD-1 level and HCC development during NA treatment (Table 3).

T cells play an important role in chronic HBV infection because persistent exposure to high concentrations of viral antigens leads to various degrees of T-cell functional impairment, termed T-cell exhaustion. ${ }^{17,23}$ PD-1 is expressed on the surface of activated T cells ${ }^{28}$ and has two ligands, PD-L1 and PD-L2. ${ }^{29}$ The binding of PD-1 to its ligands may inhibit activated T cells and plays a critical role in T-cell exhaustion. ${ }^{30}$ Therefore, PD-1 expression is correlated with the exhaustion of T cells in chronic infections and cancers. ${ }^{31}$ Soluble forms of PD-1 have been detected in blood. Unlike PD-1, the function of sPD-1 is unclear. However, sPD-1 can compete with PD-1 by binding to PD-L1 and blocking PD-1/PD-L1 interactions, enhancing antigen-specific T-cell immunity and dendritic cell maturation, ${ }^{32}$ restore the proliferative response of virus-specific CD4 and CD8 T cells during chronic infection, ${ }^{33,34}$ and enhance antitumor responses. ${ }^{35}$

Previous studies have reported relationships between the serum sPD-1 level and clinical/virological characteristics in patients with chronic HBV infection. ${ }^{22,36,37}$ According to these reports, the serum sPD-1 level is significantly positively correlated with the HBV-DNA and ALT levels and similar between cases 
of non-cirrhosis and cirrhosis, consistent with our findings (Fig. 3). The serum SPD-1 level in patients with non-cirrhosis, cirrhosis, and HCC was significantly higher than that in asymptomatic carriers. Patients with chronic HBV infection and HCC have the highest SPD-1 level. Xia et al. reported that the serum SPD-1 level decreased gradually after NA treatment in most cases, ${ }^{36}$ consistent with our results. Interestingly, the serum SPD-1 level did not increase again upon HCC development during NA treatment (Fig. 4), although increased SPD-1 reflects an immune response to HBV infection or HCC. These findings suggest that NA treatment reduces the immune response to HBV infection and $\mathrm{HCC}$ by suppressing HBV replication and HCC development, and on-treatment SPD-1 is not useful as a biomarker for identifying HCC during NA treatment.

Chang et al. performed a retrospective study of 120 patients with HCC receiving radical resection and showed that a low level of SPD-1 at baseline was correlated with poor overall survival as well as a trend toward shortened disease-free survival in patients with HBV-related $\mathrm{HCC} .{ }^{24}$ These findings suggest that a low SPD-1 level worsens the prognosis of HCC. This is consistent with our result that a lower SPD-1 level was a risk factor for HCC development during NA treatment in patients with chronic HBV infection.

Cheng et al. showed that a high sPD-1 level $(>282 \mathrm{pg} / \mathrm{mL})$ at baseline was associated with a twofold increase in the risk of HCC in a cohort of HBV patients. ${ }^{23}$ By contrast, our findings showed that patients with chronic HBV infection with a lower SPD-1 level $(\leq 447.27 \mathrm{pg} / \mathrm{mL})$ at baseline had a higher risk of developing HCC during NA treatment. This discrepancy could be attributed to the difference in the background of the study population between Cheng et al.'s and our studies. The study population in Cheng et al. consisted of asymptomatic carriers not on NA treatment; the serum SPD-1 level in asymptomatic carriers is significantly lower than in patients with chronic HBV infection. ${ }^{22}$ In our study, serum SPD-1 was detected only in the analysis limited to patients treated with NAs; NAs are administered to a large number of patients with chronic HBV infection, who may have a higher risk of HCC. In addition, we speculate that the impact of competition with PD-1 for binding to PD-L1 and blocking PD-1/PD-L1 interactions was reduced in patients with chronic HBV infection with a lower SPD-1 level, leading to a reduced antitumor response. This is consistent with a previous report. ${ }^{35}$ Hence, our findings imply that patients with a lower sPD-1 level before the initiation of NA treatment should be followed up carefully as a population at higher risk of $\mathrm{HCC}$, despite a report that NA treatment for chronic HBV infection reduces the risk of HCC development. ${ }^{2-4}$

Our study had some limitations. First, it was a single-center study conducted on a relatively small scale. Second, most of the study population consisted of patients with HBV genotype $C$, and it is unclear whether the results can be extended to patients with different HBV genotypes because HBV genotype $\mathrm{C}$ is associated with a higher risk of HCC development. Therefore, further large-scale studies with patients with various HBV genotypes are necessary.

In conclusion, our findings suggest that SPD-1 is a novel predictive biomarker for HCC development during NA treatment. 


\section{Declarations}

Acknowledgments: This work was supported by JSPS KAKENHI grant number JP18K15792 and the Program for Basic and Clinical Research on Hepatitis of the Japan Agency for Medical Research and Development (JP21fk0210084). We are grateful to Ms. Rie Yasuda, Ms. Ayano Fujikawa, and Ms. Sanae Deguchi for collecting the data.

Authors' contributions: R.K. and M.E. designed research; all authors performed research; R.K. analyzed data; R.K. wrote the main manuscript text; all authors reviewed the manuscript.

Competing interests: In the past year, Dr. Sawako Uchida-Kobayashi has received research funding from Bristol-Myers. The other authors declare that they have no financial conflicts of interest.

\section{References}

1. European Association for the Study of the Liver. EASL 2017 Clinical Practice Guidelines on the management of hepatitis B virus infection. J. Hepatol, 67, 370-398 (2017).

2. Liaw, Y. F. et al. Lamivudine for patients with chronic hepatitis B and advanced liver disease. N. Engl. J. Med, 351, 1521-1531 (2004).

3. Hiramatsu, N., Yamada, R. \& Takehara, T. The suppressive effect of nucleos(t)ide analogue treatment on the incidence of hepatocellular carcinoma in chronic hepatitis B patients. J. Gastroenterol. Hepatol, 31, 546-552 (2016).

4. Hosaka, T. et al. Long-term entecavir treatment reduces hepatocellular carcinoma incidence in patients with hepatitis B virus infection. Hepatology, 58, 98-107 (2013).

5. Yamada, R. et al. Impact of alpha-fetoprotein on hepatocellular carcinoma development during entecavir treatment of chronic hepatitis B virus infection. J. Gastroenterol, 50, 785-794 (2015).

6. Di, M. V. et al. Clinical outcome of HBeAg-negative chronic hepatitis B in relation to virological response to lamivudine. Hepatology, 40, 883-891 (2004).

7. Lim, Y. S. et al. Mortality, liver transplantation, and hepatocellular carcinoma among patients with chronic hepatitis B treated with entecavir vs lamivudine. Gastroenterology, 147, 152-161 (2014).

8. Kozuka, R. et al. Association between HLA-DQA1/DRB1 polymorphism and development of hepatocellular carcinoma during entecavir treatment. J. Gastroenterol. Hepatol, 34, 937-946 (2019).

9. Thimme, R. et al. CD8(+) T cells mediate viral clearance and disease pathogenesis during acute hepatitis B virus infection. J. Virol, 77, 68-76 (2003).

10. Boni, C. et al. Characterization of hepatitis B virus (HBV)-specific T-cell dysfunction in chronic HBV infection. J. Virol, 81, 4215-4225 (2007).

11. Phillips, S. et al. CD8(+) T cell control of hepatitis B virus replication: direct comparison between cytolytic and noncytolytic functions. J. Immunol, 184, 287-295 (2010). 
12. Park, J. J. et al. Hepatitis B virus-specific and global T-cell dysfunction in chronic hepatitis B. Gastroenterology. 150, 684-695. e5(2016).

13. Zhang, H. H. et al. Regulatory T cells in chronic hepatitis B patients affect the immunopathogenesis of hepatocellular carcinoma by suppressing the anti-tumour immune responses. J. Viral. Hepat, 17, 34-43 (2010).

14. Dinney, C. M. et al. Regulation of HBV-specific CD8(+) T cell-mediated inflammation is diversified in different clinical presentations of HBV infection. J. Microbiol, 53, 718-724 (2015).

15. Ye, B. et al. T-cell exhaustion in chronic hepatitis B infection: current knowledge and clinical significance. Cell. Death. Dis, 6, e1694 (2015). https://www.nature.com/articles/cddis201542

16. Streeck, H. et al. Antigen load and viral sequence diversification determine the functional profile of HIV-1-specific CD8 + T cells. PLoS. Med, 5, e100 (2008). https://journals.plos.org/plosmedicine/article?id=10.1371/journal.pmed.0050100

17. Fisicaro, P. et al. Antiviral intrahepatic T-cell responses can be restored by blocking programmed death-1 pathway in chronic hepatitis B. Gastroenterology, 138, 682-693 (2010).

18. Gu, D., Ao, X., Yang, Y., Chen, Z. \& Xu, X. Soluble immune checkpoints in cancer: production, function and biological significance. J. Immunother. Cancer, 6, 132 (2018).

19. Dong, M. P. et al. Clinical significance of circulating soluble immune checkpoint proteins in sorafenibtreated patients with advanced hepatocellular carcinoma. Sci. Rep, 10, 3392 (2020). https://www.nature.com/articles/s41598-020-60440-5

20. Li, F. et al. Highly elevated soluble Tim-3 levels correlate with increased hepatocellular carcinoma risk and poor survival of hepatocellular carcinoma patients in chronic hepatitis B virus infection. Cancer. Manag. Res, 10, 941-951 (2018).

21. Zhao, Q. et al. The paradoxical changes of membrane and soluble herpes virus entry mediator in hepatocellular carcinoma patients. J. Gastroenterol. Hepatol, 32, 1520-1524 (2017).

22. Li, N. et al. Circulating soluble programmed death-1 levels may differentiate immune-tolerant phase from other phases and hepatocellular carcinoma from other clinical diseases in chronic hepatitis $B$ virus infection. Oncotarget, 8, 46020-46033 (2017).

23. Cheng, H. Y. et al. Circulating programmed death-1 as a marker for sustained high hepatitis $B$ viral load and risk of hepatocellular carcinoma. PLoS. One, 9, e95870 (2014). https://journals.plos.org/plosone/article?id=10.1371/journal.pone.0095870

24. Chang, B. et al. The correlation and prognostic value of serum levels of soluble programmed death protein 1 (sPD-1) and soluble programmed death-ligand 1 (SPD-L1) in patients with hepatocellular carcinoma. Cancer. Immunol. Immunother, 68, 353-363 (2019).

25. Liu, Q. et al. Soluble cytotoxic T-lymphocyte antigen 4: a favorable predictor in malignant tumors after therapy. Onco. Targets. Ther, 10, 2147-2154 (2017).

26. Finkelmeier, F. et al. High levels of the soluble programmed death-ligand (SPD-L1) identify hepatocellular carcinoma patients with a poor prognosis. Eur. J. Cancer, 59, 152-159 (2016). 
27. Han, X. et al. Pre-treatment serum levels of soluble programmed cell death-ligand 1 predict prognosis in patients with hepatitis B-related hepatocellular carcinoma. J. Cancer. Res. Clin. Oncol, 145, 303312 (2019).

28. Wherry, E. J. et al. Molecular signature of CD8 + T cell exhaustion during chronic viral infection. Immunity. 27,670-684(2007).

29. Latchman, Y. et al. PD-L2 is a second ligand for PD-1 and inhibits T cell activation. Nat. Immunol, 2, 261-268 (2001).

30. Barber, D. L. et al. Restoring function in exhausted CD8 T cells during chronic viral infection. Nature, 439, 682-687 (2006).

31. Rao, M. et al. -PD-1/PD-L1 therapy for infectious diseases: learning from the cancer paradigm. Int. J. Infect. Dis, 56, 221-228 (2017).

32. Song, M. Y., Park, S. H., Nam, H. J., Choi, D. H. \& Sung, Y. C. Enhancement of vaccine-induced primary and memory CD8(+) T-cell responses by soluble PD-1. J. Immunother, 34, 297-306 (2011).

33. Onlamoon, N. et al. Soluble PD-1 rescues the proliferative response of simian immunodeficiency virus-specific CD4 and CD8 T cells during chronic infection. Immunology, 24, 277-293 (2008).

34. Amancha, P. K., Hong, J. J., Rogers, K., Ansari, A. A. \& Villinger, F. In vivo blockade of the programmed cell death-1 pathway using soluble recombinant PD-1-Fc enhances CD4 + and CD $8+T$ cell responses but has limited clinical benefit. J. Immunol, 191, 6060-6070 (2013).

35. He, L. et al. Blockade of B7-H1 with SPD-1 improves immunity against murine hepatocarcinoma. Anticancer. Res, 25, 3309-3313 (2005).

36. Xia, J. et al. Profiles of serum soluble programmed death-1 and programmed death-ligand 1 levels in chronic hepatitis B virus-infected patients with different disease phases and after anti-viral treatment. Aliment. Pharmacol. Ther, 51, 1180-1187 (2020).

37. Zhou, L. et al. Soluble programmed death-1 is a useful indicator for inflammatory and fibrosis severity in chronic hepatitis B. J. Viral. Hepat, 26, 795-802 (2019).

38. Huang, D. Q., Lim, S. G. \& Hepatitis, B. Who to treat? A critical review of international guidelines. Liver. Int, 40, 5-14 (2020).

39. Drafting Committee for Hepatitis Management Guidelines and the Japan Society of Hepatology. Japan Society of Hepatology Guidelines for the Management of Hepatitis B Virus Infection: 2019 update. Hepatol. Res, 50, 892-923 (2020).

40. Bedossa, P. \& Poynard, T. An algorithm for the grading of activity in chronic hepatitis C. Hepatology, 24, 289-293 (1996).

41. Odagiri, N. et al. Early Change in the Plasma Levels of Circulating Soluble Immune Checkpoint Proteins in Patients with Unresectable Hepatocellular Carcinoma Treated by Lenvatinib or Transcatheter Arterial Chemoembolization. Cancers (Basel). 12, 2045; https://www.mdpi.com/2072$6694 / 12 / 8 / 2045$ (2020). 


\section{Tables}

Due to technical limitations, table 1 to 3 is only available as a download in the Supplemental Files section.

\section{Figures}

\section{(A) sICOS}

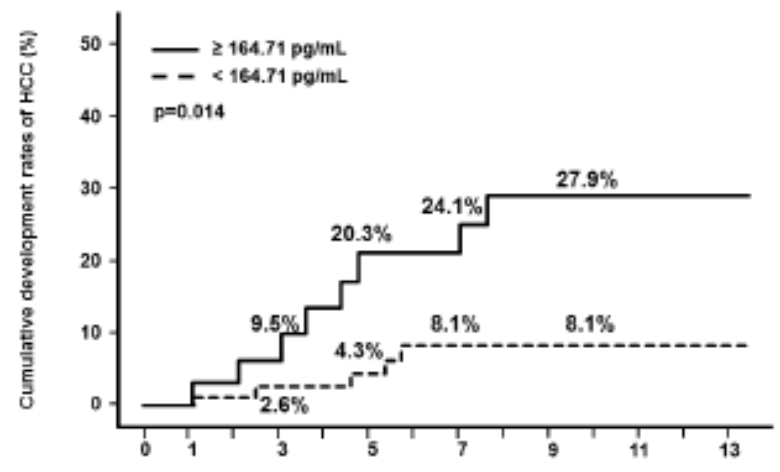

Number at risk

\begin{tabular}{|l|lllll|}
\hline Trestment duration (yr) & 0 & 3 & 5 & 7 & 10 \\
\hline $2164.71 \mathrm{pg} \mathrm{mL}$ & 39 & 27 & 22 & 21 & 11 \\
\hline$<164.71 \mathrm{pg} / \mathrm{mL}$ & 89 & 70 & 55 & 35 & 16 \\
\hline
\end{tabular}

(C) SCD40

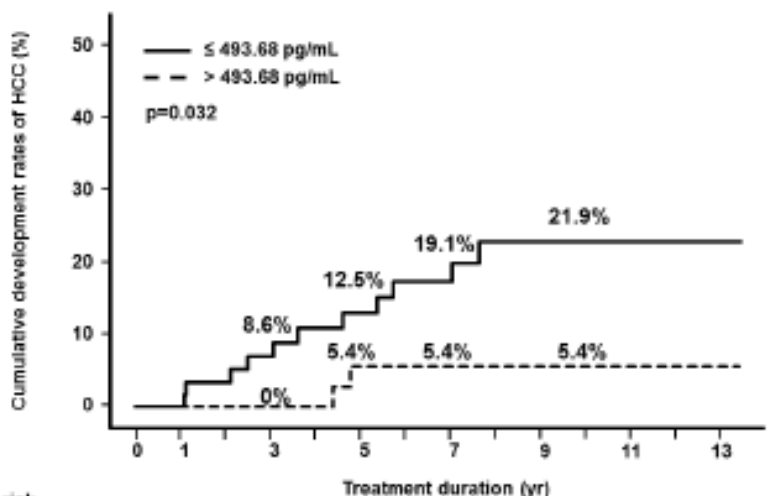

Number at risk

\begin{tabular}{|l|lllll|}
\hline Trestment duration (yr) & 0 & 3 & 5 & 7 & 10 \\
\hline $3499.68 \mathrm{pg} \mathrm{mL}$ & 64 & 52 & 43 & 34 & 14 \\
\hline$>493.68 \mathrm{pg} / \mathrm{mL}$ & 58 & 45 & 34 & 22 & 12 \\
\hline
\end{tabular}

(B) SPD-1

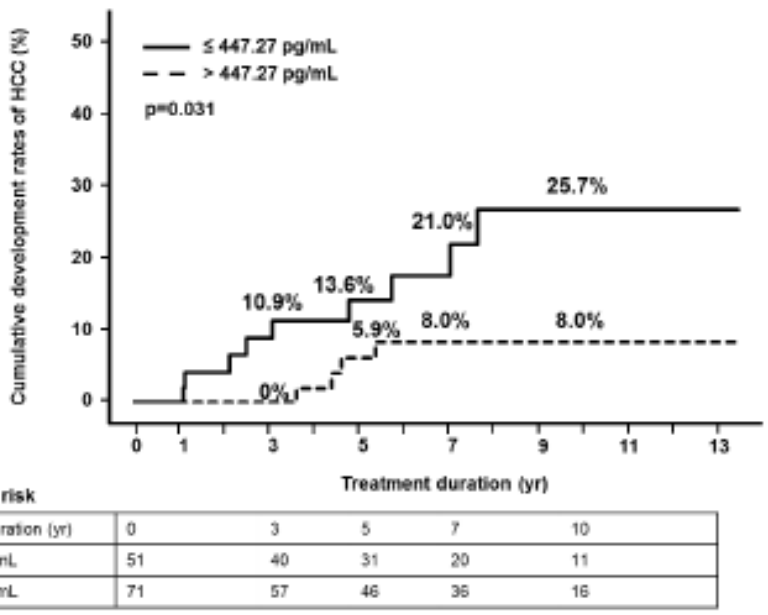

(D) SHVEM

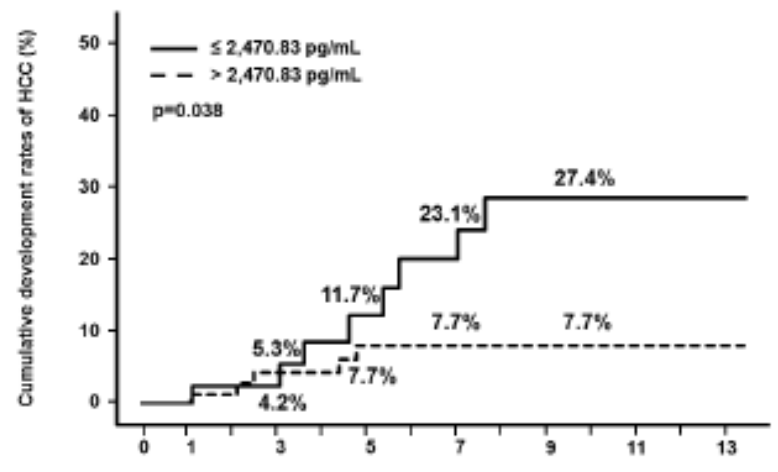

Number at risk

Treatment duration (yr)

\begin{tabular}{|l|lllll|}
\hline Treatmerk duration $(y \mathrm{y})$ & 0 & 3 & 5 & 7 & 10 \\
\hline$\leq 2.470 .83 \mathrm{pg} / \mathrm{mL}$ & 43 & 34 & 25 & 21 & 10 \\
\hline$>2,470.83 \mathrm{pg} \mathrm{mL}$ & 79 & 63 & 52 & 35 & 17 \\
\hline
\end{tabular}

\section{Figure 1}

Cumulative rates of hepatocellular carcinoma ( $\mathrm{HCC}$ ) according to $(\mathrm{A})$ soluble inducible T cell costimulator (sICOS), (B) soluble programmed cell death-1 (sPD-1), (C) soluble CD40 (sCD40), and (D) soluble herpes virus entry mediator (sHVEM) levels at baseline. 


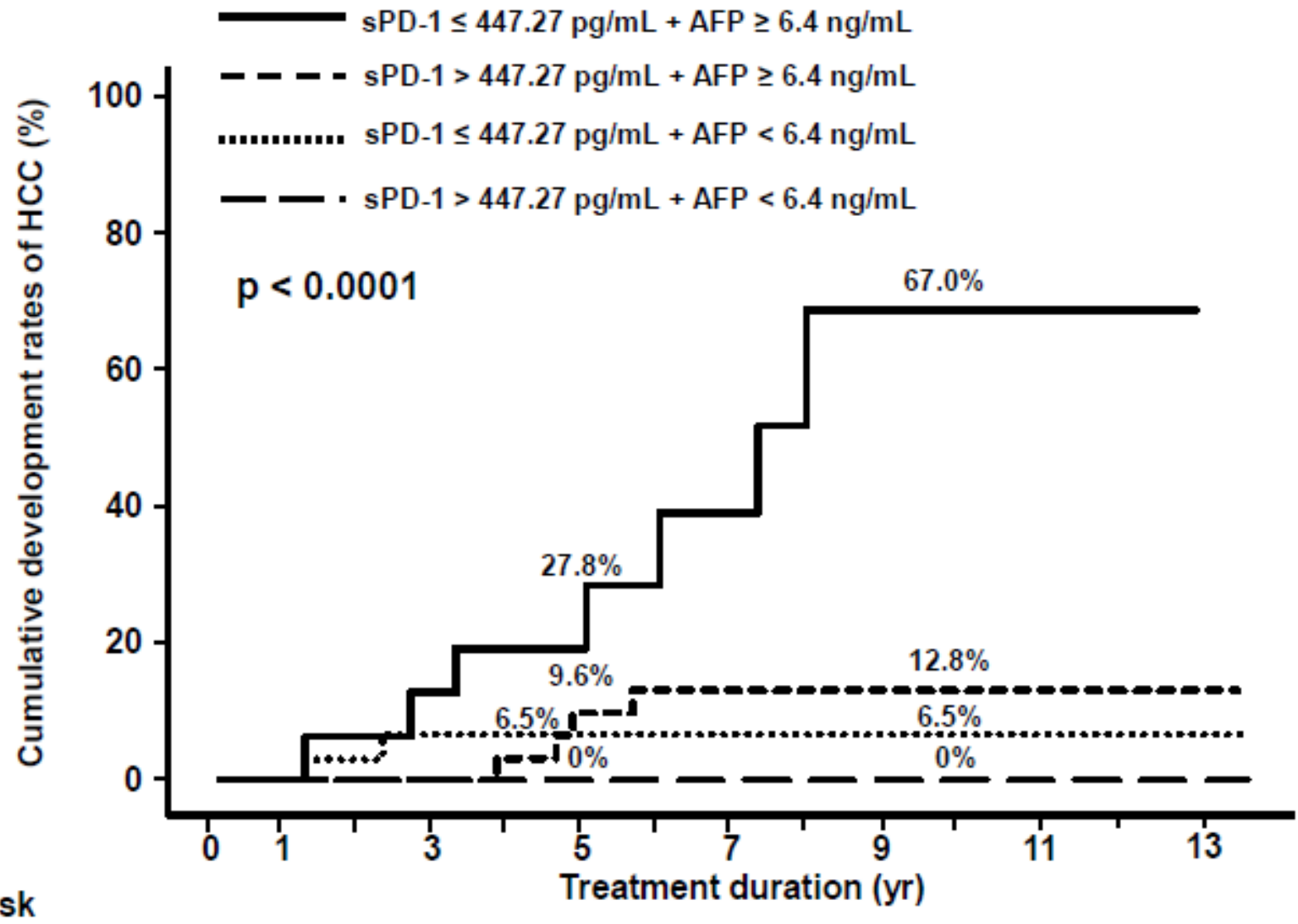

Number at risk

\begin{tabular}{|l|lllll|}
\hline Treatment duration (yr) & 0 & 3 & 5 & 7 & 10 \\
\hline sPD-1 $\leq 447.27+$ AFP $\geq 6.4$ & 17 & 14 & 9 & 5 & 2 \\
\hline sPD-1 $>447.27+$ AFP $\geq 6.4$ & 41 & 34 & 29 & 24 & 11 \\
\hline sPD-1 $\leq 447.27+$ AFP $<6.4$ & 34 & 27 & 23 & 16 & 10 \\
\hline sPD-1 $>447.27+$ AFP $<6.4$ & 30 & 24 & 18 & 13 & 6 \\
\hline
\end{tabular}

Figure 2

Cumulative rates of HCC development according to the combination of serum SPD-1 and a-fetoprotein (AFP) levels. 
(A) HBV-DNA

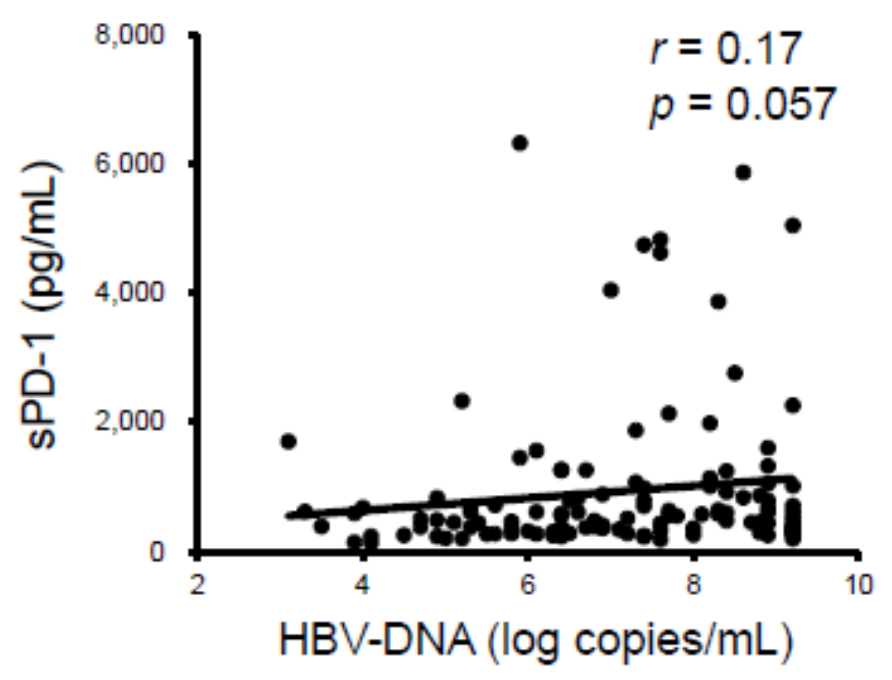

(C) Liver fibrosis

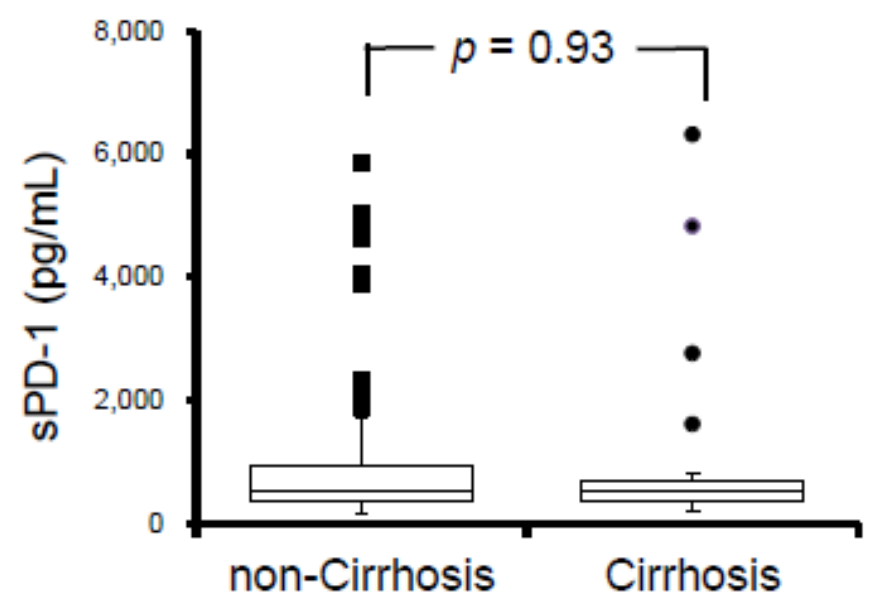

(B) ALT

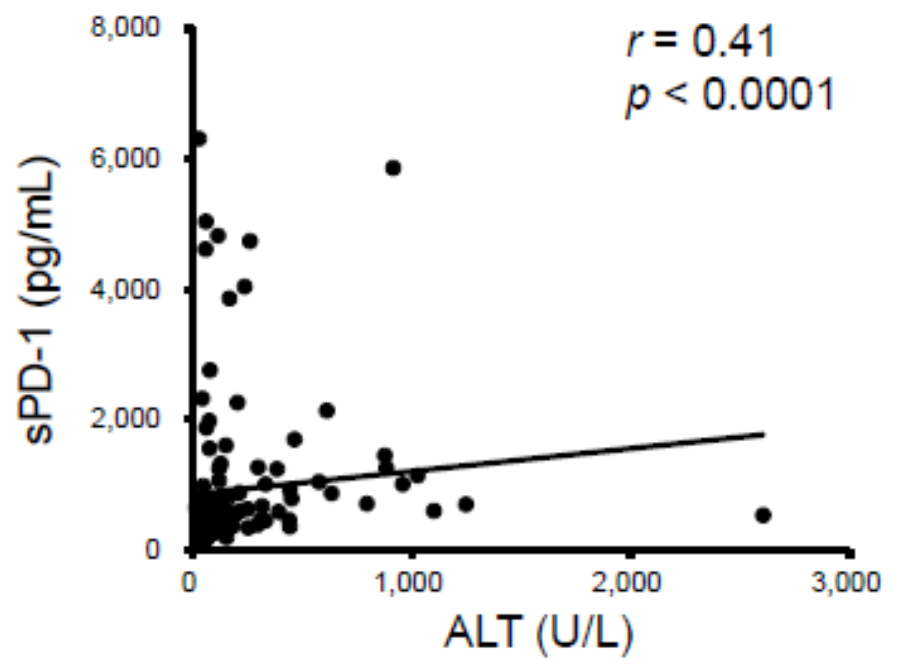

Figure 3

Correlation between the serum SPD-1 level and (A) hepatitis B virus (HBV)-DNA and (B) alanine aminotransferase (ALT) levels. Serum SPD-1 levels in non-cirrhotic and cirrhotic patients (C). 


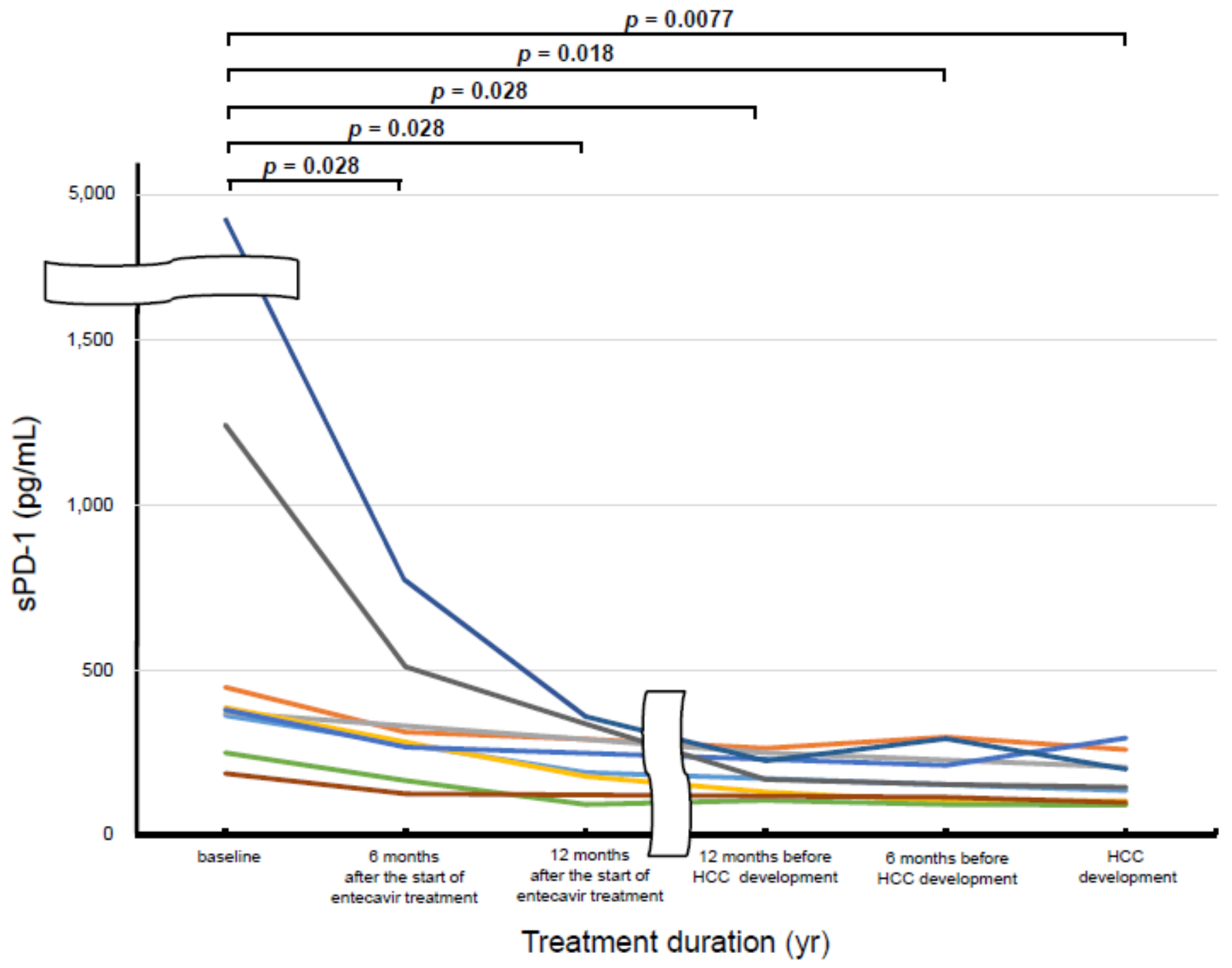

Figure 4

Dynamic changes in the serum SPD-1 level in patients who developed HCC during entecavir treatment.

\section{Supplementary Files}

This is a list of supplementary files associated with this preprint. Click to download.

- Table1.doc

- Table2.doc

- Table3.doc

- SupplementaryFigure1.pdf

- SupplementaryFigure2.pdf 